\title{
Effects of miR-26a on the expression of Beclin 1 in retinoblastoma cells
}

\author{
M. Li ${ }^{1,2}$, X.M. Chen', D.M. Wang' ${ }^{2}$ L. Gan² and Y. Qiao ${ }^{2}$ \\ ${ }^{1}$ Department of Ophthalmology, West China Hospital of Sichuan University, \\ Chengdu, Sichuan, China \\ ${ }^{2}$ Department of Ophthalmology, AVIC 363 Hospital of Chengdu, Chengdu, \\ Sichuan, China \\ Corresponding author: X.M. Chen \\ E-mail: chenxmwchsu@126.com
}

Genet. Mol. Res. 15 (1): gmr.15028193

Received August 8, 2015

Accepted November 26, 2015

Published July 15, 2016

DOI http://dx.doi.org/10.4238/gmr.15028193

\begin{abstract}
The aim of this study was to evaluate the effects of miR$26 \mathrm{a}$ on Beclin 1 expression in retinoblastoma (RB) cell lines (Y79 and WERi-RB-1). RB cells were transfected with miR-26a mimic, antagomir-26a, or control mimic. The Beclin 1 mRNA and protein levels were detected by quantitative polymerase chain reaction and western blot, respectively. The activity of Beclin 13 '-UTR reporter gene was detected with the luciferase assay. After transfection with miR-26a mimic, Beclin $1 \mathrm{mRNA}$ and protein levels as well as the activity of the 3'-UTR reporter gene decreased. However, all were increased upon inhibition of miR-26a with antagomir-26a. Beclin 1 is the target of miR-26a in human RB cell lines Y79 and WERi-RB-1, and miR-26a inhibits the expression of Beclin 1 by reducing its mRNA and protein levels.
\end{abstract}

Key words: miR-26a; Beclin-1; Retinoblastoma 


\section{INTRODUCTION}

Retinoblastoma $(\mathrm{RB})$ is the most common primary malignant intraocular tumor among children who, if untreated, will die within 1-2 years (Shields et al., 2013). The pathogenesis is closely associated with the low-density expression of anti-oncogenes (Kalsoom et al., 2015). As small non-coding endogenous RNA molecules consisting of 18-25 nucleotides, microRNAs (miRNAs or miRs) bind target mRNA by complete or incomplete pairing. They participate in many biological processes such as embryo development, cell proliferation, differentiation, apoptosis, and lipid metabolism (Slezak-Prochazka et al., 2010). Commonly regarded as an antioncogene, miR-26a regulates cell cycle in the G1 phase by inhibiting the expression of enhancer of zeste homolog 2 after transcription (Ciarapica et al., 2009). miR-26a can arrest hepatocellular carcinoma cells in the G1 phase by directly downregulating cyclins D2 and E2 (Kota et al., 2009), but its role in RB remains unknown. Autophagy, which is programmed cell survival, plays a key role in tumor progression and treatment (Ji et al., 2015). Autophagy functions differently in various tumors or stages of progression. For instance, it promotes the growth of pancreatic cancer cells but inhibits that of breast cancer cells (Yang et al., 2011). As the first discovered gene that specifically participates in autophagy in mammals, Beclin 1 predominantly affects tumor onset and progression by regulating the activity of autophagy (Liang et al., 1999). In this study, we evaluated the influence of miR-26a on expression of Beclin 1 in RB cell lines Y79 and WERi-RB-1 with the aim of identifying any potential inhibitory effects of miR-26a.

\section{MATERIAL AND METHODS}

\section{Reagents and apparatus}

Plasmids for miR-26a mimic and miR-26a antagomir were purchased from Dharmacon (USA). Beclin 1 3'-UTR lenti-reporter-luciferase vector was bought from Sigma-Aldrich (USA). Human RB cell lines Y79 and WERi-RB-1 were obtained from Stem Cell Bank, Chinese Academy of Sciences (China). Fetal bovine serum, RPMI-1640, and HBSS culture medium were provided by Gibco (MD, USA). TRIzol kit and FITC fluorescent secondary antibody were purchased from Life Technologies (CA, USA). Onestep reverse transcription-polymerase chain reaction (RT-PCR) kit was bought from Qiagen (CA, USA). Mouse anti-human GAPDH and Beclin 1 monoclonal antibodies and enhanced chemiluminescence (ECL) reagent were obtained from Santa Cruz (USA). PCR primers were synthesized by our group and BGI Biotechnology Co., Ltd. (Shenzhen, China). Primers for GAPDH: upstream, 5'-GGTGAAGGTCGGAGTCAACGG-3', and downstream, 5'-GGTCATGAGTCCTTCCACGATACC-3'. Primers for Beclin 1: upstream, 5'-ATCCTGGACCGTGTCACCATCCAGG-3', and downstream, 5'-GTTGAGCTGAGTGTCCAGCTGG-3'. The LightCycler 3.0 PCR System was provided by Bio-Rad (USA). The light microscope and inverted microscope were purchased from SANYO (Japan). UV-vis spectrophotometer was bought from Beckman Coulter (USA).

\section{Cell culture}

Human RB cells Y79 and WERi-RB-1 were cultured in RPMI-1640 containing 10\% 
fetal bovine serum and incubated in $5 \% \mathrm{CO}_{2}$ at $37^{\circ} \mathrm{C}$. The culture medium was refreshed every 3-4 days, and all cells were within the 20th passage.

\section{Experimental grouping and cell transfection}

One day before transfection, Y79 and WERi-RB-1 cells were inoculated onto 24-well plates at a density of $4 \times 10^{5} / \mathrm{mL}$ and divided into four groups: miR-26a mimic group, control mimic group, antagomir-26a group, and control antagomir group. Then, they were cultured with antibiotic-free culture medium until $90-95 \%$ confluence. DNA $(0.8 \mu \mathrm{g})$ was added to 50 $\mu \mathrm{L}$ serum-free Opti-MEM, and $2.0 \mu \mathrm{L}$ Lipofectamine ${ }^{\mathrm{TM}} 2000$ (Life Technologies) was added to another $50 \mu \mathrm{L}$ serum-free Opti-MEM I and incubated at room temperature for $5 \mathrm{~min}$. The two reagents were then mixed and incubated at room temperature for $20 \mathrm{~min}$, after which they were added onto the plates to culture the cells at $37^{\circ} \mathrm{C}$ in $5 \% \mathrm{CO}_{2}$ for $24 \mathrm{~h}$.

\section{Detection of Beclin 1 mRNA levels by RT-PCR}

The supernatant was discarded $24 \mathrm{~h}$ after transfection, and total RNA was extracted after adding $1 \mathrm{~mL}$ TRIzol reagent according to the manufacturer instructions. Based on the primers described above, the RT-PCR system was prepared according to the manufacturer instructions of the one-step RT-PCR kit. The reaction parameters were: reverse transcription at $50^{\circ} \mathrm{C}$ for $30 \mathrm{~min}$; reaction at $95^{\circ} \mathrm{C}$ for $15 \mathrm{~min}$; and cycling ( 30 cycles) with denaturation at $94^{\circ} \mathrm{C}$ for $30 \mathrm{~s}$, annealing at $57^{\circ} \mathrm{C}$ for $30 \mathrm{~s}$, and extension at $72^{\circ} \mathrm{C}$ for $30 \mathrm{~s}$. A final extension was conducted at $72^{\circ} \mathrm{C}$ for $10 \mathrm{~min}$. PCR products were subjected to agarose gel electrophoresis and photographed. Absorbance of the DNA bands was analyzed, and relative mRNA levels are reported as the ratio of absorbance of Beclin 1 to GAPDH.

\section{Detection of Beclin 1 protein expression by western blot}

The supernatant was discarded $48 \mathrm{~h}$ after transfection, and pre-cooled cell lysate buffer [ $50 \mathrm{mM}$ Tris- $\mathrm{HCl}, 150 \mathrm{mM} \mathrm{NaCl}, 0.02 \%$ sodium azide, $0.1 \%$ sodium dodecyl sulfate (SDS), $1 \%$ octylphenoxypolyethoxyethanol (NP-40), $0.5 \%$ deoxysodium cholate, $100 \mu \mathrm{g} / \mathrm{mL}$ phenylmethylsulfonyl fluoride, and $1 \mu \mathrm{g} / \mathrm{mL}$ aprotinin] was added. Then the solution was placed on ice for $20 \mathrm{~min}$ and centrifuged at $4^{\circ} \mathrm{C}$ and $12,000 \mathrm{~g}$ for $15 \mathrm{~min}$. Subsequently, the supernatant was resolved using 10\% SDS-PAGE and electronically transferred to a nitrocellulose membrane that was blocked at room temperature for $2 \mathrm{~h}$, incubated overnight with diluted GAPDH and Beclin 1 antibodies at $4^{\circ} \mathrm{C}$, and then incubated with horseradish peroxidase-labeled secondary antibodies at room temperature for $2 \mathrm{~h}$. The blot was color-developed with ECL reagent, exposed to X-ray film, developed, and fixed. After absorbance scan and image analysis, the relative protein expression level was determined and reported as the ratio of absorbance of Beclin 1 to GAPDH.

\section{Luciferase assay}

Passive lysis buffer (5X) was added into 4-times volume of distilled water and mixed. Stop \& Glo substrate $(50 X ; 2.1 \mathrm{~mL})$ was added into $105 \mathrm{~mL}$ Stop \& Glo buffer and shaken for $10 \mathrm{~s}$. After removal of the culture medium, the cells were washed with 1X PBS that was then 
removed. Afterwards, an appropriate amount of $1 \mathrm{X}$ PBS was added onto the plates that were thereafter gently shaken at room temperature for $15 \mathrm{~min}$. The lysate was transferred to a test tube containing $100 \mu \mathrm{L}$ luciferase assay reagent II to carry out the luciferase assay.

\section{Statistical analysis}

All data were analyzed by SPSS11.0 (Chicago, IL, USA), obtained by at least three independent experiments, and reported as means \pm standard deviation. Intra-group comparisons were performed with analysis of variance, and inter-group comparisons were conducted by the $t$-test. $\mathrm{P}<0.05$ was considered statistically significant.

\section{RESULTS}

\section{Beclin 1 mRNA expression detected by RT-PCR}

miR-26a was closely associated with the 3'-UTR sequence of Beclin 1, so we postulated that Beclin 1 was the target of miR-26a in humans (Figure 1). In the cells transfected with miR-26a mimic and antagomir-26a, miR-26a levels were negatively correlated with Beclin 1 levels (i.e., Beclin 1 mRNA levels decreased when miR-26a levels increased). Compared with the negative control groups, the differences were statistically significant $(\mathrm{P}<0.05$; Figure 2$)$.

\section{3'-ucccgcuaauugaccGUAUAAc-5' hsa-miR-26a 5'-agcugcugacugucgCGUAUUUu-3' Beclin 1}

Figure 1. Correlation between miR-26a and 3'-UTR sequence of Beclin 1.
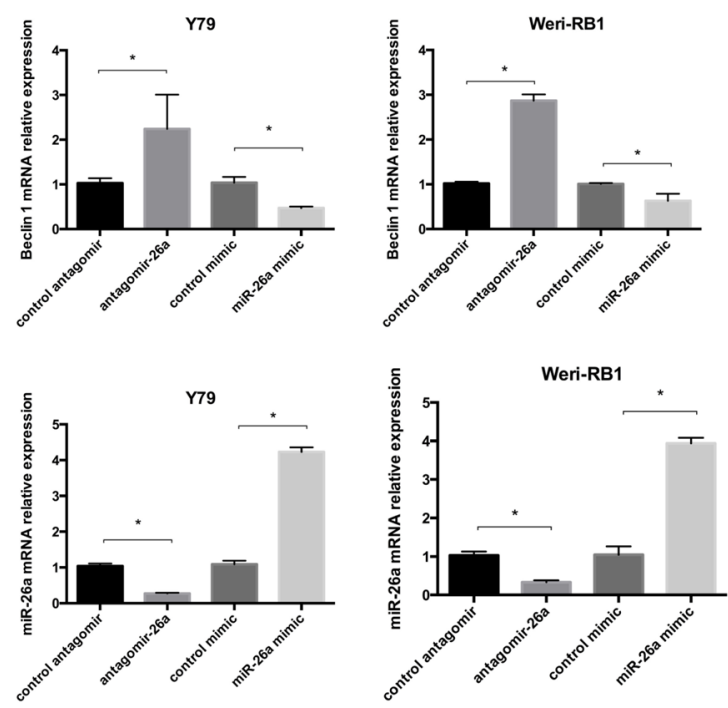

Figure 2. Beclin 1 and miR-26a mRNA levels in cells transfected with miR-26a mimic, control mimic, or antagomir-26a as detected by RT-PCR. *P $<0.05$. 


\section{Luciferase assay results}

After transfection of the cells with miR-26a mimic and antagomir-26a, the activity of the 3'-UTR reporter gene was detected by luciferase assay. The cells transfected with miR-26a mimic had significantly weakened luciferase activity, but those transfected with antagomir26a had significantly enhanced activity $(\mathrm{P}<0.05)$ compared to those of the control groups (Figure 3).
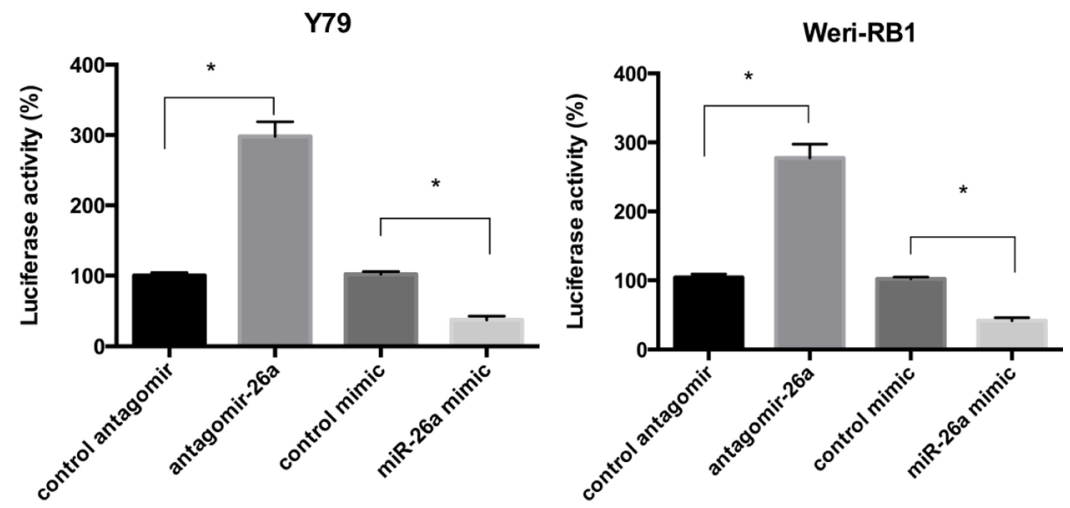

Figure 3. Luciferase activities of cells transfected with miR-26a mimic, control mimic, or antagomir-26a. *P $<0.05$.

\section{Beclin 1 protein expression detected by western blot}

Western blotting was performed after transfection with miR-26a mimic and antagomir26a. Beclin 1 protein expression, which decreased in the cells transfected with miR-26a mimic, significantly increased in those transfected with antagomir-26a (Figure 4).

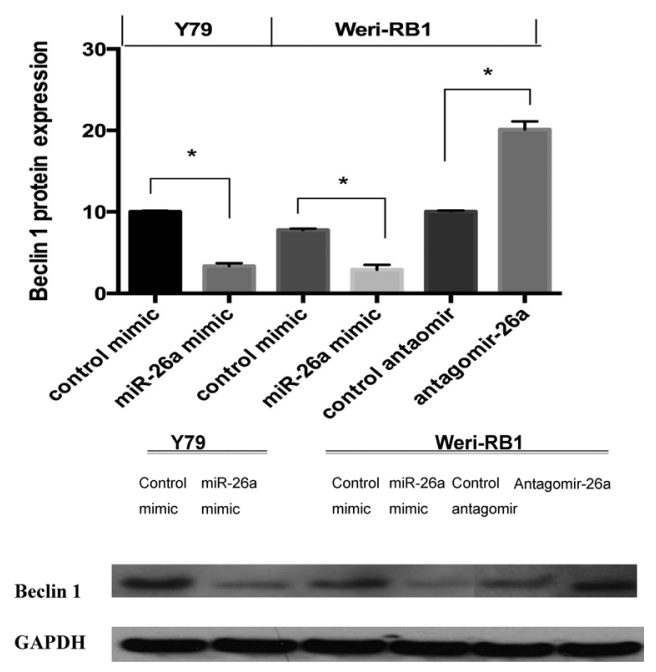

Figure 4. Beclin 1 protein expression in cells transfected with miR-26a mimic, control mimic, or antagomir-26a as detected by western blot. $* \mathrm{P}<0.05$. 


\section{DISCUSSION}

Widely present in the metabolic process of eukaryotic cells, autophagy refers to the self-protection of cells by the degradation of proteins and organelles through lysosomes to maintain survival, renewal, material reuse, and homeostasis (Levine and Kroemer, 2008). It has previously been shown that autophagic death occurred upon apoptotic pathway deficiency, but now autophagy is commonly regarded as programmed cell survival. Autophagy plays a key role in tumor progression and treatment (Livesey et al., 2009). In various tumor types or at different stages of tumor progression, autophagy functions distinctly differently. For example, it promotes the growth of pancreatic cancer cells but inhibits that of breast cancer cells (Yang et al., 2011; Frassanito et al., 2015). As one of the anti-oncogenes, Beclin 1 is related to tumor onset and progression. It is prone to single-allele deletion/mutation in 40-75\% of human sporadic breast, ovarian, and prostate cancer cells (Cuervo and Dice, 1996; 2000). Knockout of the Beclin 1 allele in mice increases the probability of spontaneous malignant transformation, and they are prone to hepatitis B virus-induced pre-malignant lesions $(\mathrm{Qu}$ et al., 2003; Yue et al., 2003).

Many of the regulatory effects of miRNAs on autophagy have been studied. Autophagy requires proteins encoded by autophagy-related genes (ATGs). miRNA-101 can inhibit the autophagy of breast cancer cells by regulating the expression of ATGs, especially that of ATG4D (Frankel et al., 2011). In addition, Beclin 1 expression in breast and lung cancer cells can be suppressed by miR-30a (Zhu et al., 2009). miRNAs interact with regulatory elements at mRNA 3'-UTRs through seed sequences at the 5'-end, thereby inhibiting mRNA expression. It is well-documented that chromosome aberration is mainly responsible for the changes in miRNA expression in human tumors. As an anti-oncogene, miR-26a regulates cell cycle in the G1 phase by inhibiting the expression of enhancer of zeste homolog 2 after transcription. In addition, miR-26a directly downregulates the expression of cyclins D2 and E2 in liver cancer cells, thus arresting them in the G1 phase (Kota et al., 2009). Being associated with the survival and apoptosis of many types of cells, miR-26a facilitates cell proliferation and decreases apoptosis. miR-26a can enhance the proliferation and angiogenesis of glial cell tumors (Qian et al., 2013), as well as suppress the apoptosis of mouse skeletal muscle cells and promote their differentiation and proliferation (Dey et al., 2012). Besides functioning by inhibiting mRNA expression, miRNAs also participate in the regulation of cell activities, including autophagy, so we linked them with autophagyregulating protein Beclin 1.

Since there are corresponding sites in the seed sequence of miR-26a and the 3'UTR of Beclin 1, Beclin 1 is one of the regulatory targets of miR-26a. To further prove the relationship between miR-26a and Beclin 1, we assessed the influence of miR-26a on Beclin 1 expression in human RB cells. When the expression level of miR-26a was elevated by transfection with miR-26a mimic, Beclin 1 mRNA levels and reporter gene activity significantly decreased. Conversely, both increased when the cells were transfected with antagomir-26a. Beclin 1 protein expression, which was reduced after transfection with miR26a mimic, increased after antagomir-26a treatment. The findings demonstrate that miR-26a inhibits Beclin 1 expression in human RB cells. Beclin 1 is a crucial anti-apoptotic and proautophagy protein, so we will endeavor to verify whether miR-26a regulates the autophagy of RB cells through Beclin 1. 


\title{
Conflicts of interest
}

\author{
The authors declare no conflict of interest.
}

\section{REFERENCES}

Ciarapica R, Russo G, Verginelli F, Raimondi L, et al. (2009). Deregulated expression of miR-26a and Ezh2 in rhabdomyosarcoma. Cell Cycle 8: 172-175. http://dx.doi.org/10.4161/cc.8.1.7292

Cuervo AM anad Dice JF (1996). A receptor for the selective uptake and degradation of proteins by lysosomes. Science 273: 501-503.

Cuervo AM and Dice JF (2000). Regulation of lamp2a levels in the lysosomal membrane. Traffic 1: 570-583. http://dx.doi. org/10.1034/j.1600-0854.2000.010707.x

Dey BK, Gagan J, Yan Z and Dutta A (2012). miR-26a is required for skeletal muscle differentiation and regeneration in mice. Genes Dev. 26: 2180-2191. http://dx.doi.org/10.1101/gad.198085.112

Frankel LB, Wen J, Lees M, Høyer-Hansen M, et al. (2011). microRNA-101 is a potent inhibitor of autophagy. EMBO J. 30: 4628-4641. http://dx.doi.org/10.1038/emboj.2011.331

Frassanito MA, De Veirman K, Desantis V, Marzo LD, et al. (2015). Halting prosurvival autophagy by TGFbeta inhibition in bone marrow fibroblasts overcomes bortezomib resistance in multiple myeloma patients. Leukemia October: 21; Epub ahead of print.

Ji Y, Di W, Yang Q, Lu Z, et al. (2015). Inhibition of autophagy increases proliferation inhibition and apoptosis induced by the PI3K/mTOR inhibitor NVP-BEZ235 in breast cancer cells. Clin. Lab. 61: 1043-1051.

Kalsoom S, Wasim M, Afzal S, Shahzad MS, et al. (2015). Alterations in the RB1 gene in Pakistani patients with retinoblastoma using direct sequencing analysis. Mol. Vis. 21: 1085-1092.

Kota J, Chivukula RR, O’Donnell KA, Wentzel EA, et al. (2009). Therapeutic microRNA delivery suppresses tumorigenesis in a murine liver cancer model. Cell 137: 1005-1017. http://dx.doi.org/10.1016/j.cell.2009.04.021

Levine B and Kroemer G (2008). Autophagy in the pathogenesis of disease. Cell 132: 27-42. http://dx.doi.org/10.1016/j. cell.2007.12.018

Liang XH, Jackson S, Seaman M, Brown K, et al. (1999). Induction of autophagy and inhibition of tumorigenesis by beclin 1. Nature 402: 672-676. http://dx.doi.org/10.1038/45257

Livesey KM, Tang D, Zeh HJ and Lotze MT (2009). Autophagy inhibition in combination cancer treatment. Curr. Opin. Investig. Drugs 10: 1269-1279.

Qian X, Zhao P, Li W, Shi ZM, et al. (2013). MicroRNA-26a promotes tumor growth and angiogenesis in glioma by directly targeting prohibitin. CNS Neurosci. Ther. 19: 804-812.

Qu X, Yu J, Bhagat G, Furuya N, et al. (2003). Promotion of tumorigenesis by heterozygous disruption of the beclin 1 autophagy gene. J. Clin. Invest. 112: 1809-1820. http://dx.doi.org/10.1172/JCI20039

Shields CL, Fulco EM, Arias JD, Alarcon C, et al. (2013). Retinoblastoma frontiers with intravenous, intra-arterial, periocular, and intravitreal chemotherapy. Eye (Lond.) 27: 253-264. http://dx.doi.org/10.1038/eye.2012.175

Slezak-Prochazka I, Durmus S, Kroesen BJ and van den Berg A (2010). MicroRNAs, macrocontrol: regulation of miRNA processing. RNA 16: 1087-1095. http://dx.doi.org/10.1261/rna.1804410

Yang S, Wang X, Contino G, Liesa M, et al. (2011). Pancreatic cancers require autophagy for tumor growth. Genes Dev. 25: 717-729. http://dx.doi.org/10.1101/gad.2016111

Yue Z, Jin S, Yang C, Levine AJ, et al. (2003). Beclin 1, an autophagy gene essential for early embryonic development, is a haploinsufficient tumor suppressor. Proc. Natl. Acad. Sci. USA 100: 15077-15082. http://dx.doi.org/10.1073/ pnas. 2436255100

Zhu H, Wu H, Liu X, Li B, et al. (2009). Regulation of autophagy by a beclin 1-targeted microRNA, miR-30a, in cancer cells. Autophagy 5: 816-823. http://dx.doi.org/10.4161/auto.9064 